LA-7942-MS
Informal Report
W. 3078

Sampling the Fermi-Dirac Density

to

$\frac{7}{\omega}$

(1)

$\frac{2}{\mathrm{c}}$ 
LA-7942-MS

Informal Report

vC-32

Issued: July 1979

\title{
Sampling the Fermi-Dirac Density
}

\author{
E. D. Cashwell \\ C. J. Everett
}

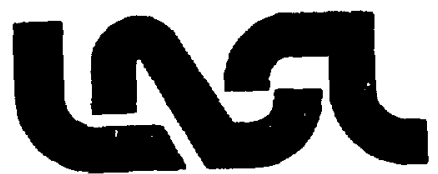

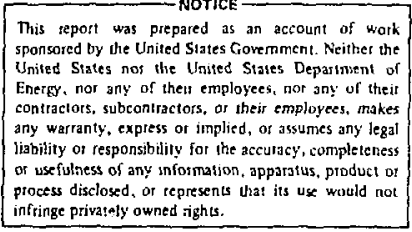


SAMPLING THE FERMI-DIRAC DENSITY

by

E. D. Cashwell and C. J. Everett

\section{ABSTRACT}

A method is given for sampling the nonre1ativistic Fermi-Dirac electron energy density for all values of the "degeneracy parameter" $n$ on the range $-\infty<n \leqslant 50$. The efficiency of the various rejection techniques employed is never less than $30 \%$, and drops below $50 \%$ only for a short range of $n$ values around $n=2$. The range can certainly be extended beyond $\eta=50$, the efficiency there being $71 \%$, and decreasing very slowly.

\section{THE FERMI-DIRAC DENSITY}

The nonrelativistic Fermi-Dirac density for the electron velocity (Ref. 1 , p. 333) is given by

$$
\begin{aligned}
& P\left(v_{x}, v_{y}, v_{z}\right) d v_{x} d v_{y} d v_{z}=\frac{2 m^{3}}{n h^{3}} \Phi(E) d v_{x} d v_{y} d v_{z}, \\
& \Phi(E)=1 /\left[\exp \left(\frac{E}{9}-\eta\right)+1\right],
\end{aligned}
$$

where $\mathrm{m}=9.1096 \times 10^{-28} \mathrm{~g}$ is the electron mass, $\mathrm{n}$ is the number of electrons per $\mathrm{cm}^{3}, h=6.6262 \times 10^{-27}$ erg sec is Planck's constant, $E=\frac{1}{2} \mathrm{mv}^{2}$ erg is the electron energy, with $v^{2}=v_{x}^{2}+v_{y}^{2}+v_{z}^{2}$, and $\theta=k T$ erg is the "temperature," $\mathrm{k}=1.3806 \times 10^{-16} \mathrm{erg} /{ }^{\circ} \mathrm{K}$ being the Boltzmann constant. In Eq. (2), $n$ is the "degeneracy parameter," depending on $\mathrm{n}$ and $\theta$ in such a way as to make

$$
\int_{-\infty}^{\infty} \int_{-\infty}^{\infty} \int_{-\infty}^{\infty} p\left(v_{x}, v_{y}, v_{z}\right) d v_{x} d v_{y} d v_{z}=1 .
$$


If $Q(v, \theta, \phi)$ denotes the corresponding "spherical coordinate density," then $\mathrm{Q}(v, \theta, \phi) \mathrm{d} v \mathrm{~d} \theta \mathrm{d} \phi=\frac{2 \mathrm{~m}^{3}}{n h^{3}} \Phi(\mathrm{E})\left(v^{2} \sin \theta\right) \mathrm{d} v \mathrm{~d} \theta \mathrm{d} \phi$, and the speed density is, therefore, given by

$$
q(v) d v=\frac{8 \pi m^{3}}{n h^{3}} \Phi(E) v^{2} d v, 0<v<\infty
$$

Since $E=\frac{3}{2} m v^{2}$, we obtain for the energy density

$f(E) d E=q(v) \frac{d v}{d E} d E=\frac{8 \sqrt{2} \pi m^{3 / 2}}{n h^{3}} E^{\frac{1}{2}} \Phi(E) d E, 0<E<\infty$

Setting $y=E / \theta$, the $y$ density is seen to be

$$
\begin{aligned}
p(y) d y & =f(E) \frac{d E}{d y} d y=\frac{8 \sqrt{2} \pi m^{3 / 2} e^{3 / 2}}{n h^{3}} \cdot \frac{y^{\frac{1}{2}} d y}{e^{y-n}+1} \\
& \equiv C^{-1} \frac{y^{\frac{1}{2}} d y}{e^{y-n}+1}, 0<y<\infty .
\end{aligned}
$$

From the necessary relation

$$
\int_{0}^{\infty} p(y) d y=1
$$

it follows that the $n, 9$-dependent parameter $n$ must be determined so that

$$
I(n) \equiv \int_{0}^{\infty} \frac{y^{\frac{1}{2}} d y}{e^{y-n}+1}=\frac{h^{3}}{8 \sqrt{2} \pi m^{3 / 2}} \cdot \frac{n}{\theta^{3 / 2}} \equiv c .
$$

It is easy to verify that $I(n)$ and $I^{\prime}(n)$ are positive

for $-\infty<n<\infty$, while 


$$
\begin{aligned}
& I(\eta)=\int_{0}^{\infty} \frac{y^{\frac{1}{2}} e^{-y} d y}{e^{-\eta}+e^{-y}}<\frac{\Gamma(3 / 2)}{e^{-\eta}} \rightarrow 0 \text { as } \eta \rightarrow-\infty, \\
& I(n)>\int_{0}^{\eta} \frac{y^{\frac{1}{2}} d y}{e^{y-n}+1}>\int_{0}^{n} \frac{y^{\frac{1}{2}} d y}{2}=\frac{n^{3 / 2}}{3} \rightarrow \infty \text { as } n \rightarrow \infty .
\end{aligned}
$$

Thus $I(n)$ strictly increases from $I(-\infty)=0$ to $I(\infty)=\infty$, and for every $C>0$ in Eq. (8), there is a unique $n$ on $(-\infty, \infty)$ such that $I(n)=c$.

Values of $I(\eta)$ have been tabulated (Ref. 2,3) at intervals of 0.1 for $-4 \leqslant n \leqslant 20$. Table I below gives an idea of the variation of $I(n)$ on this range.

TABLE I

THE FUNCTION I $(\eta)$

\begin{tabular}{|c|c|}
\hline$\eta$ & $I(\eta)$ \\
\hline-4 & .016128 \\
-3 & .043366 \\
-2 & .114588 \\
-1 & .290501 \\
0 & .678094 \\
1 & 1.39638 \\
2 & 2.50246 \\
2.5 & 3.1966 \\
3 & 3.97699 \\
4 & 5.77073 \\
5 & 7.83797 \\
6 & 10.1443 \\
7 & 12.6646 \\
8 & 15.3805 \\
9 & 18.2776 \\
10 & 21.3445 \\
11 & 24.5718 \\
12 & 27.9518 \\
13 & 31.4775 \\
14 & 35.1430 \\
15 & 38.9430 \\
16 & 42.8730 \\
17 & 46.9286 \\
18 & 51.1061 \\
19 & 55.4019 \\
20 & 59.8128 \\
\hline & \\
\hline
\end{tabular}

Outside of these limits the following approximations are recommended (Ref. 3):

$$
\begin{aligned}
& I(n) \cong \frac{\sqrt{\pi}}{2} \mathrm{e}^{\eta}, n<-4, \\
& I(\eta) \cong \frac{2}{3} n^{3 / 2}, n>20 .
\end{aligned}
$$

One may note that $\frac{\sqrt{\pi}}{2} \mathrm{e}^{-4}=.016232$, while $\frac{2}{3}(20)^{3 / 2}=59.628$, as compared with the values $I(-4)$ and $I(20)$ in TABLE I

\section{PRELIMINARY DETERMINATION OF C} AND $n$

For a given density $\mathrm{n}$ and temperature $\theta=\mathrm{kT}$, one first computes the value of

$$
\begin{aligned}
& \mathrm{C}=\frac{\mathrm{h}^{3}}{8 \sqrt{2} \pi \mathrm{m}^{3 / 2}} \cdot \frac{\mathrm{n}}{\theta^{3 / 2}} \\
& \cong 1.835 \times 10^{-16} \frac{\mathrm{n}}{\mathrm{T}^{3 / 2}}
\end{aligned}
$$


Guided by the above remarks we then determine the corresponding $\eta_{l}$ as follows:

(a) If $\mathrm{C}<.016128, n$ is given by $\mathrm{e}^{n}=\frac{2}{\sqrt{\pi}} \mathrm{C}$,

(b) if $.016128 \leqslant C \leqslant 59.8128, \eta$ is found from the tables cited (Ref. 2, 3),

(c) if $\mathrm{C}>59.8128$, then $\eta=(3 \mathrm{C} / 2)^{2 / 3}$.

For the known values of $C>0$ and $n$ on $(-\infty, \infty)$ one must now sample the density

$$
p(y)=c^{-1} \frac{y^{\frac{1}{2}}}{e^{y-n}+1}, 0<y<\infty
$$

for $y>0$, and set the energy $E=\theta y$. Due to the curious nature of the function $p(y)$ we are forced to use two different methods depending on the value of $\eta$.

III. THE CASE $(-\infty<n \leqslant 5 / 2)$

For a value of $\eta \leqslant 5 / 2$, we write

$$
\begin{aligned}
p(y) d y & =C^{-1} \Gamma(3 / 2) e^{n} \cdot \frac{y^{\frac{1}{2}} e^{-y} d y}{\Gamma(3 / 2)} \cdot \frac{1}{1+e^{n-y}} \\
& \equiv A^{-1} \cdot p_{1}(y) d y \cdot h(y),
\end{aligned}
$$

where $p_{1}(y)$ is the density

$$
p_{1}(y)=y^{\frac{1}{2}} \mathrm{e}^{-y} / \Gamma(3 / 2), 0<y<\infty,
$$

$h(y)$ is the "acceptance factor"

$$
0<h(y)=1 /\left(1+e^{n-y}\right)<1,0<y<\infty,
$$

and the efficiency of the corresponding rejection technique is

$$
\mathrm{A}=2 \mathrm{C} / \sqrt{\pi} \mathrm{e}^{n}
$$

Following the usual method $(\operatorname{Re} f .4, R 7)$, we sample $p_{1}(y)$ for $y$ on $(0, \infty)$, and accept $y$ with probability $h(y)$. 
The density $p_{1}(y)$ is easily sampled (Ref. 4, C 32); a brief indication of the routine follows:

Sampling $y^{\frac{1}{2}} e^{-y} / \Gamma(3 / 2)$ for $y$ on $(0, \infty)$.

1. Generate random numbers, $r_{1}, r_{2}$ on $(0,1)$.

2. Is $S=r_{1}^{2}+r_{2}^{2} \leqslant 1$ ? Yes (advance to (3)), No (return to (1)).

3. Set $\mu_{i}=-\frac{\log S}{S} \cdot r_{i}^{2}, i=1,2$.

4. Generate nexi random number $r$.

5. Set $y=-\log r+\mu_{i}$.

(Two samples of $y$ are obtained, which may be used successively.)

The efficiency $A$ in Eq. (16), based on the values of $C=I(\eta)$ in TABLE $I$, are listed for various values of $\eta \leqslant 3$ in TABLE II. Note that, for the recommended approximation $e^{\eta}=2 \mathrm{C} / \sqrt{\pi}(n<-4)$, the efficiency $A$ appears to be 1 , although, of course, there may be some rejection.

In principle, the above method applies for all $n$. We have drawn the line at $\eta=5 / 2$ simply because the method of the next part is relatively easy to apply for $n>5 / 2$, as will appear. If enough trouble were taken in finding the minimum $h$ in part $I V$, the $n$ dividing line could be pushed to the left, with a resulting increase in efficiency.

IV. THE CASE $(n>5 / 2)$.

We first note that the function

$$
p(y)=c^{-1} \frac{y^{\frac{1}{2}}}{e^{y-n}+1}
$$

TABLE I I

EFFICIENCY A

\begin{tabular}{|c|c|}
\hline$n$ & $\mathrm{~A}$ \\
\hline-4 & .99 \\
-3 & .98 \\
-2 & .96 \\
-1 & .89 \\
0 & .77 \\
1 & .58 \\
2 & .38 \\
2.5 & .30 \\
3 & .22 \\
\hline
\end{tabular}

is decreasing for $y>\eta$, provided $\eta$ exceeds 1. For, an easy computation shows that the inequality $p^{\prime}(y)<0$ follows from the relation $(2 y-1) e^{y-n}$ $>(2 \eta-1) e^{0}>1$.

If we define

$$
A_{1}=\int_{0}^{\eta} p(y) d y, A_{2}=1-A_{1} \text {, }
$$


we may sample $A_{1}^{-1} p(y)$ dy on $(0, \eta)$ with probability $A_{1}$, and $A_{2}^{-1} p(y)$ dy on $(n, \infty)$ with probability $\mathrm{A}_{2}$ (Ref. 4, C 3 ).

(a) The first of these is simple, for we may write

$$
A_{1}^{-1} p(y) d y=A_{1}^{-1} C^{-1}\left((2 / 3) n^{3 / 2}\right)\left(e^{-n}+1\right)^{-1} \cdot \frac{y^{\frac{1}{2}} d y}{(2 / 3) n^{3 / 2}} \cdot \frac{e^{-n}+1}{e^{y-n}+1}
$$

Thus we easily sample the density $y^{\frac{1}{2}} /\left((2 / 3) n^{3 / 2}\right)$ for $y=n r^{2 / 3}$ on $(0, n)$, accepting $y$ with probability $\left(e^{-n}+1\right) /\left(e^{y-n}+1\right)<1$, the efficiency of the technique being

$$
\varepsilon_{1}=3 A_{1} C\left(e^{-n}+1\right) / 2 n^{3 / 2} .
$$

This certainly exceeds $\frac{1}{2}$. For,

$$
A_{1}=\int_{0}^{n} c^{-1} \frac{y^{\frac{1}{2}} d y}{e^{y-n}+1}>c^{-1} \frac{\left((2 / 3) n^{3 / 2}\right)}{2}=\frac{c^{-1} n^{3 / 2}}{3}
$$

Thus $3 A_{1} C>n^{3 / 2}$ and $E_{1}>\frac{1}{2}$ (for any $n>0$ ).

Note that the value of $A_{1}$ is irrelevant for the rejection technique, except insofar as it enters into the efficiency $\varepsilon_{1}$. However, $A_{1}$ is required for the probabilities in Eq. (17).

It is clear that

$$
\begin{aligned}
& A_{1}=B / C, \\
& \text { where } B \equiv \int_{0}^{n} \frac{y^{\frac{1}{2}} d y}{e^{y-i 7}+1} .
\end{aligned}
$$

Hence the value of $B$ as well as $C$ is required for each $n \geqslant 5 / 2$ which arises. These values are listed for $\eta=3,4, \ldots, 50$ in TABLE III, and were obtained by numerical integration using Simpson's rule. It may be helpful to include the following routine. 
Simpson's method for $B=\int_{0}^{n} \frac{y^{\frac{1}{2}} d y}{e^{y-n}+1}, n=3,4, \ldots, 50$.

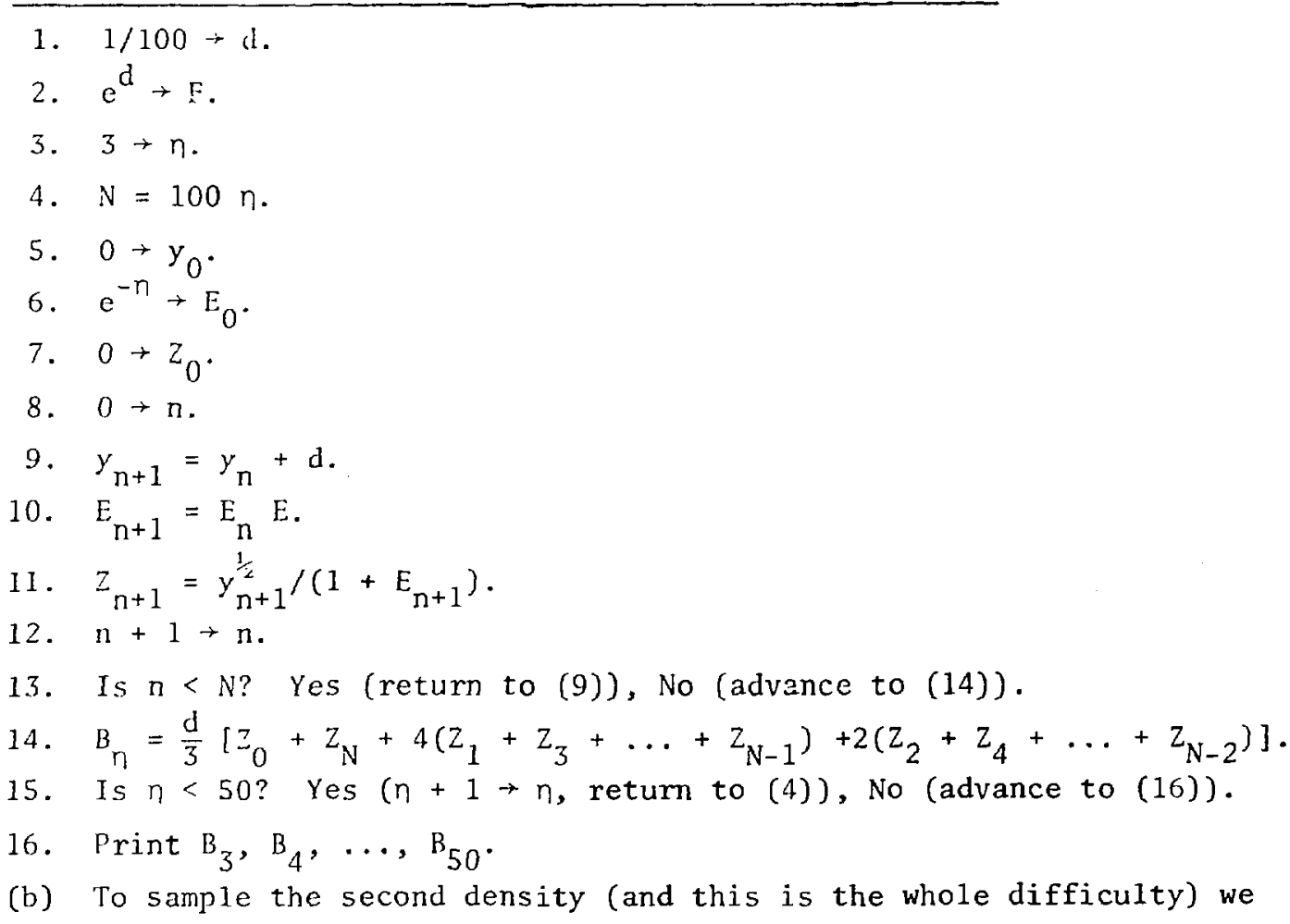
write

$$
A_{2}^{-1} p(y) d y=A_{2}^{-1} C^{-1} e^{n} \Gamma_{n} H \cdot \frac{y e^{-y} d y}{\Gamma_{n}} \cdot \frac{H^{-1}}{y^{\frac{1}{2}}\left(1+e^{n-y}\right)} \text {, }
$$

where

$$
\begin{aligned}
& H=\max 1 /\left[y^{\frac{3}{2}}\left(1+e^{n-y}\right)\right] \text { for } n<y<\infty, \\
& \Gamma_{n}=\int_{n}^{\infty} y e^{-y} d y=(n+1) e^{-n} .
\end{aligned}
$$

Hence, if we determine

$$
h=\min \left[y^{\frac{1}{2}}\left(1+e^{n-y}\right)\right] \text { for } n<y<\infty \text {, }
$$


the density (22) becomes

$$
A_{2}^{-1} C^{-1}(\eta+1) h^{-1} \cdot \frac{y e^{-y}}{\Gamma_{n}} \cdot \frac{h}{y^{\frac{1}{2}}\left(1+e^{n-y}\right)} \text {. }
$$

We propose to sample $y e^{-y} / \Gamma_{\eta}$ for $y$ on $(n, \infty)$, accepting $y$ with probability

$$
\mathrm{h} /\left[\mathrm{y}^{\frac{1}{2}}\left(1+\mathrm{e}^{n-y}\right)\right]<1 \text {, }
$$

the efficiency of the technique being now

$$
\varepsilon_{2}=A_{2} C h /(n+1) \text {. }
$$

Recalling that $A_{2}=1-A_{1}$ and $A_{1}=B / C$, this becomes

$$
\varepsilon_{2}=(C-B) h /(n+1) \text {, }
$$

where $B$ is defined by Eq. (21), and tabulated in TABLE III.

Thus the minimum value $h$ in $\mathrm{Eq}$. (25) is required not only for evaluating the efficiency $\varepsilon_{2}$, but also for the acceptance probability (27). We next turn to the determination of $h$.

For the function

$$
h(y)=y^{\frac{3}{2}}\left(1+\mathrm{e}^{n-y}\right), y \geqslant n,
$$

one may show that $h\left(r_{0}\right)=2 \eta^{\frac{1}{2}}, h(\infty)=\infty$, with

$$
h^{\prime}(y)=\left[1-(2 y-1) e^{n-y}\right] / 2 y^{\frac{1}{2}}
$$

Thus $h^{\prime}(n)<0, h^{\prime}(\infty)=0$, and the minimum of $h(y)$ occurs at some $y_{0}>n$. We therefore require the unique $y_{0}>n$ for which $h^{\prime}(y)=0$, that is to say, for which

$$
g(y) \equiv(2 y-1) e^{n-y}-1=0
$$


Now $g(\eta)=(2 \eta-1)-1>0$ since $\eta_{i}>1$, and $g(\infty)=-1$. However, it can be shown that $g(y)$ is decreasing on $(n, \infty)$ only if $n>3 / 2$, and is concave up only if $n>5 / 2$. Hence, Newton's method for the zero $y_{0}$ of $g(y)$, with an initial $y=n$, is safe only if $n>5 / 2$, and this has dictaced cur requirement $n>5 / 2$ in the present method. The values of $h$ for $\eta=3,4, \ldots, 50$ are listed in TABLE III, together with the corresponding efficiencies $\varepsilon_{2}$.

The zero $y_{0}$ and associated minimum $h$ of $h(y)$ were computed by the following routine.

Newton's method for $h=\min _{(n, \infty)}\left[y^{\frac{1}{2}}\left(1+e^{n-y}\right)\right], n=3,4, \ldots, 50$.

1. $3 \rightarrow \eta$.

2. $n \rightarrow y$.

3. $y^{\prime}=y+\frac{(2 y-1) e^{n-y}-1}{\left(2 y^{\prime \prime}-3\right) e^{n-y}}$.

4. Is $y^{\prime}-y<.001$ ? No $\left(y^{\prime} \rightarrow y\right.$, return to (3)), Yes ladvance to (5)).

5. $h_{n}=\left(y^{\prime}\right)^{\frac{1}{2}}\left(1+e^{n-y^{\prime}}\right)$.

6. Is $\eta<50$ ? Yes $(\eta+1 \rightarrow \eta$, return to (2)), No (advance to (7)).

7. Print $h_{3}, h_{4}, \ldots, h_{50}$.

It only remains to indicate how the "tail end" of the $\Gamma$-density $y e^{-y} / \Gamma_{\eta}$, $\eta<y<\infty$, is to be sampled for $y>n$. For this, we employ the ingenious method of Carey and Drijard (Ref. 5), which in our case may be formulated by the following routine.

1. Set $P=e^{-\eta}, A=e^{-\eta}(1+\eta), B=1 /(1+\eta)$.

2. Generate random numbers $\rho_{1}, \rho_{2}$ on $(0,1)$.

3. Is $o_{1} \leqslant B$ ? Yes (advance to (4)), No (advance to (5)).

4. Set $r_{1}=A \rho_{1}, r_{2}=\rho_{2}$ (advance to (6)).

5. Set $r_{1}=P \exp \left[(1+\eta) \rho_{1}-1\right], r_{2}=\rho_{2} P / r_{1}$ (advance to (6)).

6. Set $y=-\log r_{1} r_{2}$.

The justification of this is based on the remarks below.

(a) To sample the density $\mathrm{ye}^{-y} / \Gamma(2)$ on its full range $(0, \infty)$ (Ref. 4, C 22), one generates random numbers $r_{1}, r_{2}$ and sets

$$
y=-\log \mathrm{r}_{1} \mathrm{r}_{2}
$$

where $\left(r_{1}, r_{2}\right)$ may be thought of as a point uniformly distributed in the unit square. 
TABLE III

DATA FOR CASE $\eta>5 / 2$

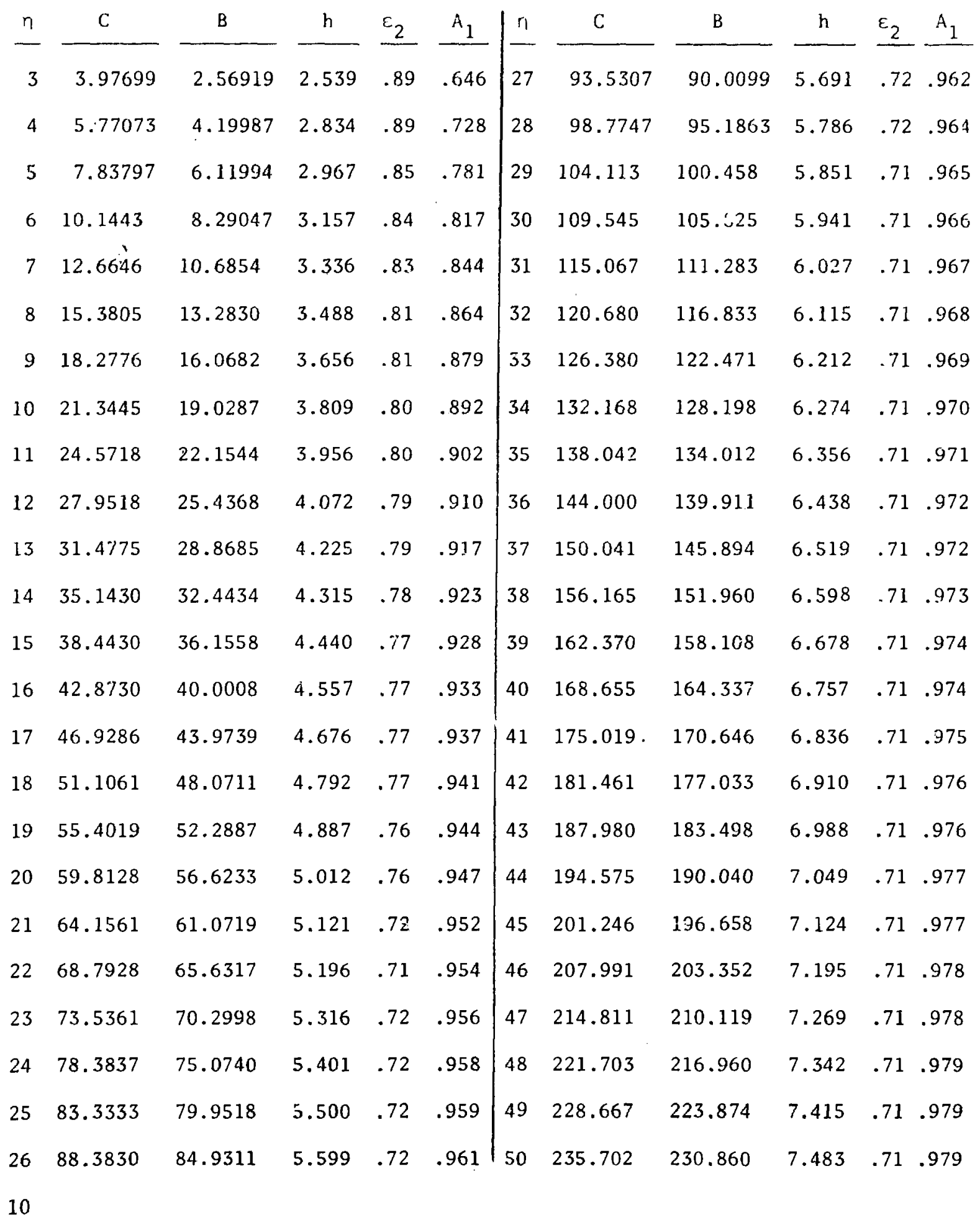


(b) But for the tail end density, one requires only such foints for which $y>\eta$, i.e., for whjch

$$
r_{1} r_{2}<e^{-r_{1}} \equiv p
$$

One could, of course, throw points $\left(r_{1}, r_{2}\right)$ uniformly in the urit square, and reject those lying above the hyperbola $r_{1} r_{2}=P$, but the efficiency would be poor.

(c) The above (nonrejection) device is valid since the two transformations in (4) and (5) both have Jacobian $e^{-\eta}(1+\eta)$, independent of $\rho_{1}, \rho_{2}$, and so transform the two rectangular areas of the full $\rho_{1}, \rho_{2}$ unit square deiermined by the line $\rho_{1}=B$ in a uniform way into the required two areas of the $r_{1}, r_{2}$ unit square; the first a rectangle of base $e^{-n}$ and height 1 , of area $e^{-n}$, and the second lying directly below the hyperbola $r_{1} r_{2}=e^{-\eta}$, with base $1-e^{-\eta}$, and area $\eta \mathrm{e}^{-\eta}$.

V. VALUE OF $I(\eta)$ FOR $\eta \leqslant 0$

For arbitrary $n>0$, and $A \equiv e^{n} \leqslant 1$, one may write

$$
\begin{aligned}
J(n) & =\int_{0}^{\infty} \frac{y^{n-1} d y}{e^{y-n}+1}=\int_{0}^{\infty} \frac{y^{n-1}\left(A e^{-y}\right) d y}{1+\left(A e^{-y}\right)} \\
& =\sum_{j=1}^{\infty}(-1)^{j+1} \int_{0}^{\infty} y^{n-1}\left(A e^{-y}\right)^{j} d y \\
& =\sum_{j=1}^{\infty} \frac{(-1)^{j+1} A^{j}}{j^{n}} \int_{0}^{\infty}(j y)^{n-1} e^{-j y} d(j y) \\
& =\left(\sum_{j=1}^{\infty}(-1)^{j+1} \frac{A^{j}}{j^{n}}\right) \int_{0}^{\infty} x^{n-1} e^{-x} d x \equiv \bar{\zeta}(A, n) \Gamma(n) .
\end{aligned}
$$

For $r_{k}=3 / 2$, this gives in our case

$$
I(n)=\bar{\zeta}(A, 3 / 2) \Gamma(3 / 2)=\frac{\sqrt{\pi}}{2} \bar{\zeta}\left(e^{n}, 3 / 2\right),
$$

determining $n$ implicitly in terms of $I(n)$.

In particular, this shows that 


$$
I(0)=\frac{\sqrt{\pi}}{2} \sum_{j=1}^{\infty} \frac{(-1)^{j+1}}{j^{3 / 2}}=\frac{\sqrt{\pi}}{2}\left(1-\frac{1}{\sqrt{2}}\right) \zeta(3 / 2)=.678094,
$$

where $\zeta$ is the Riemann zeta-function (cf. TABLE I).

In fact, a method (Ref. 4, R 8) for sampling $p(y)$ can be based on the above relations when $n<0$, but the routine of Part III seems simpler and does not restrict $\eta$ to negative values.

VI. THE MARGINAL DENSITY OF $v_{x}$

It is remarkable that, by introducing polar coordinates $r, \theta$ for $v_{y}, v_{z}$, the marginal density of $v_{x}$ on $(-\infty, \infty)$ may be obtained in the explicit form (Ref. 1 , p. 334)

$$
\begin{aligned}
P_{1}\left(v_{x}\right) & =\int_{-\infty}^{\infty} \int_{-\infty}^{\infty} P\left(v_{x}, v_{y}, v_{z}\right) d v_{y} d v_{z} \\
& =\frac{4 \pi m^{2} \theta}{n h^{3}} \log \left[1+\exp \left(n-\frac{m v_{x}^{2}}{2 \theta}\right)\right] .
\end{aligned}
$$

For $u=(m / 2 \theta)^{\frac{1}{2}} v_{x}$, we then have the u-density

$$
d(u)=(2 C)^{-1} \log \left(1+e^{n} e^{-u^{2}}\right),
$$

which seems a more well-behaved function than $p(x)$, and would, of course, serve our purpose. However, none of our attempts to sample $d(u)$ have proved feasible.

\section{REFERENCES}

1. F. W. Sears, An Introduction to Thermodynamics, the Kinetic Theory of Gases, and Statistical Mechanics (Addison-Wesley Publishing Co., Inc., Cambridge, Mass., 1953).

2. J. McDouga11, E. C. Stoner, "The Computation of Fermi-Dirac Functions," Phil. Trans. Roy. Soc. A, 237, 67 (1939).

3. A. C. Beer, M. N. Chase, P. F. Choquard, "Extension of the McDougal1-Stoner Tables of the Fermi-Dirac Functions," Helv. Phys. Acta, 28, 529 (1955).

4. C. J. Everett, E. D. Cashwel1, "A Monte Carlo Sampler," Los Alamos Scientific Laboratory report LA-5061-MS (1972). 
5. D. C. Carey, D. Drijard, "Monte Carlo Phase Space with Limited Transverse Momentum," Journal of Computational Physics, 28, 327 (197\%). 